\title{
Are "Dichotic" Deficits Uniquely Dichotic? Investigating Dichotic Performance with the Dichotic Digits Difference Test (DDdT) in a Large Clinical Population of Children Referred for an Auditory Processing Assessment
}

\author{
DOI: 10.3766/jaaa.19037
}

\author{
Sharon Cameron*† \\ Harvey Dillon* $*$ \$
}

\begin{abstract}
Background: Previous studies in a large population of typically developing (TD) children and a small clinical group showed high correlations between the dichotic and diotic conditions of the Dichotic Digits difference Test (DDdT), as well as between DDdT performance and measures of memory and attention.

Purpose: The purpose of the study was to investigate the performance on the DDdT in a large clinical sample.

Research Design: Correlational analysis between the DDdT diotic condition and the dichotic free recall (FR) right-ear, left-ear, and total (ear-averaged) conditions, as well as between DDdT and memory performance.

Study Sample: One hundred one children ( 6 years 3 months to 15 years 0 month, mean 9 years 6 months) were referred for assessment to the Australian Hearing Central Auditory Processing Disorder (CAPD) service. Results were compared with data from 112 TD children collected from previously published studies.

Data Collection and Analysis: Z-scores were used to account for the effect of age on performance. Mean differences between clinical and TD children were investigated using analysis of variance (ANOVA). Pearson product-moment correlations determined the strength of relationships between DDdT conditions and the number memory forward (NMF) and reversed (NMR) subtests of the Test of Auditory Processing Skills-Third Edition.

Results: Performance by the clinical group on the DDdT dichotic FR (RE, LE, and total) conditions was significantly correlated with the diotic condition $(r=0.7 ; 0.7,0.8 ; p<0.001)$. Significant correlations were found between the DDdT diotic and dichotic FR conditions and the NMF $(r=0.5-0.6, p<0.001)$ and NMR $(r=0.2-0.5, p<0.025-0.001)$. ANOVA revealed no significant difference between the TD and clinical groups $(p=1.0000)$ in respect to the advantage they got from dichotic listening (calculated as dichotic FR total minus diotic score). Multiple regression revealed that diotic performance and short-term memory accounted for $68 \%$ of the variation in dichotic performance. Random measurement error accounted for a further $16 \%$.
\end{abstract}

Conclusions: Factors other than dichotic performance strongly impact a child's ability to perform a dichotic digit listening task. This result has widespread implications in respect to the interpretation of CAPD test results. Kingdom

*National Acoustic Laboratories, Sydney, Australia; $†$ Macquarie University, Sydney, Australia; $\ddagger$ University of Manchester, Manchester, United

Corresponding author: Sharon Cameron, Macquarie University, Australian Hearing Hub, Macquarie University NSW 2109, Australia; Email: sharon.cameron@mq.edu.au

This research is funded by the Australian government through the Department of Health. Sharon Cameron and Harvey Dillon acknowledge the support of Macquarie University. Dr Dillon further acknowledges the support of the NIHR Manchester Biomedical Research Centre.

Parts of the research described in this article were presented at the British Society of Audiology Inaugural e-Conference, December 2017, the Audiology Australia National Conference in Sydney, Australia, May 2018, and the World Congress of Audiology in Cape Town, South Africa, November 2018. 
Key Words: central auditory processing disorder, dichotic, diotic, free recall

\begin{abstract}
Abbreviations: ARIA = Auditory Rehabilitation for Interaural Asymmetry; CANS = central auditory nervous system; CAPD = Central Auditory Processing Disorder; DDdT $=$ Dichotic Digits difference Test; FR = free recall; LE = left ear; NMF = number memory forward; NMR = number memory reversed; $\mathrm{RE}=$ right ear; $\mathrm{REA}=$ right-ear advantage; $\mathrm{RME}=$ random measurement error; $\mathrm{SD}=$ standard deviation; TAPS-3 = Test of Auditory Processing Skills - Third Edition; TD = typically developing
\end{abstract}

\section{INTRODUCTION}

A lthough the makeup of auditory processing test batteries varies widely from clinic to clinic, one of the most common types of tests used is the dichotic listening test (Martin et al, 2007; Schmithorst et al, 2013; Weihing and Atcherson, 2014; Fischer et al, 2017; Kelley and Littenberg, 2018; Magimairaja and Nagaraj, 2018). The dichotic digits tests have been reported to be a sensitive measure for detecting cortex and brain stem lesions (Musiek, 1983; Hurley and Musiek, 1997). Musiek et al (2011) investigated the sensitivity, specificity, and efficiency of four commonly used behavioral central auditory processing tests in 20 children and adults aged 13-59 years with known lesions of the central auditory nervous system (CANS). Results were compared with 29 adult controls. The experimental group performed significantly more poorly than controls on the left ear (LE) and right ear (RE) of the dichotic digits test, the frequency pattern test, and the competing sentences test. The frequency pattern test generated the highest overall sensitivity, specificity, and efficiency (90\%). Using a strict criterion (positive diagnosis determined by failure on two or more tests), a dichotic digit/frequency pattern two-test battery exceeded the specificity obtained by administering the frequency pattern test alone, albeit with slightly lower sensitivity and efficiency. The authors note, however, that the study results may not completely generalize to children and adults presenting with auditory processing deficits who have no known, specific central lesion (arguably most of the individuals assessed with the dichotic digits test).

As detailed in the introduction to Cameron et al (2016a), a number of recent studies have not only reported significant correlations between dichotic performance and cognitive ability but also cast doubt on the interpretation of uncharacteristic right-ear advantage (REA) scores as impaired conduction properties of specific auditory pathways. Instead, it is suggested results can be explained in terms of dynamic processes, including those in which individuals deliberately direct their attention in different ways (Westerhausen and Hugdahl, 2008; Hiscock and Kinsbourne, 2011; Cameron et al, 2016a,b; Stavrinos et al, 2018). Because of the wide use of dichotic tests around the world and the ongoing questions raised in respect to efficacy, the Dichotic Digits difference Test (DDdT; Cameron et al, 2013) was developed to investigate the relationship between dichotic processing and cognitive abilities via the use of differential test scores.

In the DDdT dichotic free recall (FR) condition, scores are reported for $\mathrm{LE}, \mathrm{RE}$, and total (average $\mathrm{LE}$ and $\mathrm{RE}$ ) performance. Two dichotic directed conditions (LE and $\mathrm{RE}$ ) can also be assessed. The DDdT is unique, however, because of the addition of a diotic control task. This simultaneous segregation task shares many response demands with the usual dichotic tasks. It was incorporated with the intent of differentiating children who perform below expectations because of poor dichotic processing skills from those who perform poorly because of impaired attention, memory, or other cognitive abilities. The inclusion of the diotic condition enabled calculation of a "dichotic advantage" score. Dichotic advantage is measured as the average of the FR total score minus the diotic score. This difference score provides a means of assessing genuine dichotic ability by minimizing the impact of cognitive skills on performance. REA is also calculated.

Cameron et al (2016a) detailed the development of the DDdT as well as the collection of normative and retest reliability data from ten adults and 62 children aged 7-11 years. Z-scores, which were calculated from the normative data for the baseline conditions, as well as the difference scores, provide a measure of typical performance on the DDdT. It was found that FR dichotic processing (LE, RE, and total) was highly correlated with diotic processing ( $r$ ranging from 0.5 to $0.6, p<0.0001)$.

A second study (Cameron et al, 2016b) investigated the role of cognitive abilities on DDdT test performance in a group of 50 typically developing (TD) children and ten children recruited from the Australian Hearing central auditory processing disorder (CAPD) service who were diagnosed with a memory or dichotic deficit. Again, it was found that diotic performance was significantly correlated with performance on all the DDdT dichotic FR conditions $(r=0.6-0.8 ; p<0.00001)$. Furthermore, significant correlations were found between the dichotic and diotic conditions, and the cognitive measures of attention and memory, with $r$ ranging from 0.4 to 0.5 ( $p<0.01-0.001)$. Indeed, $61 \%$ of the variance in FR total scores for the children in this study was accounted for by factors that do not involve the 
perception of dichotic stimuli. The DDdT dichotic advantage measure was investigated in the subset of clinical children and found to aid in differentiating true dichotic from spurious results. All but one child in the clinical group had deficits on the DDdT that could be explained by deficits in other processes (Cameron and Dillon, 2018). It was concluded that a larger clinical study with the DDdT was warranted.

Since the publication of Cameron et al (2016a,b), further research on the efficacy of dichotic testing has been published. Using a medical diagnostic framework, Kelley and Littenberg (2018) assessed a variety of peerreviewed literature and test documentation for evidence of reliability, accuracy, usefulness, and value of 11 dichotic tests (with stimuli consisting of dichotically aligned digits, consonants, or words). Based on the framework criteria, it was concluded that the data available did do not support the routine use of dichotic tests of binaural integration and recommended discontinuing the use of such tests for clinical evaluation of children.

Moore et al (2018) evaluated the auditory processing assessment records of 1,113 children aged 5-19 years who had normal pure-tone audiometry and middleear pathology. Assessments included the dichotic digits tests. Ninety percent of those evaluated had one or more additional difficulties, with $>40 \%$ having attention deficit hyperactivity disorder. Participants were divided into three groups based on diagnosis (APD, weakness, and undiagnosed). In respect to dichotic testing, left-ear advantage (EA) was nearly as common as right EA, and as there was no difference in EA between the three groups ( $p \geq 0.10$ ), EA was therefore considered a weak predictor of diagnosis. Moore et al (2018) concluded that based on both their analysis, as well as the results published by Cameron et al (2016a,b), “... interpretation of dichotic test results does not appear to provide a clear diagnostic structure, other than a general problem selectively attending to and recalling simultaneously presented stimuli" (p. 165), and that this finding may suggest a need for both further cognitive assessment, and a relevant solution, for example, remediation through targeted cognitive training.

Magimairaja and Nagaraj (2018) analyzed the literature concerning the relationships between working memory, attention, and auditory processing (including dichotic test performance). It was stated that attention, an integral component of working memory, is suggested to be primarily responsible for the strong association between working memory and complex cognitive task performance. However, in respect to auditory processing ability, the authors noted that the direction of causality remains elusive and the influence of attention potentially varies depending on the nature and demands of the particular auditory processing task. In a discussion on the clinical implications arising from the literature review-which included Cameron et al (2016a,b) Magimairaja and Nagaraj (2018) recommended that clinicians and researchers move toward the hierarchical assessment method detailed in Dillon et al (2012) and Cameron et al (2015). Effective integration of cognitivelinguistic factors in addition to the auditory processing assessment was stipulated. For example, as detailed in the Cameron et al (2015) study of 666 children assessed for auditory processing by the Australian Hearing CAPD service, short-term and working memory is assessed before the commencement of any dichotic testing. Magimairaja and Nagaraj (2018) note that such a decision tree approach is not only time and cost-effective but also can lead to the determination of target areas that may benefit from intervention. The authors also recommend the use of auditory processing assessments like the DDdT that use differential test scores to minimize language and cognitive influences on test performance.

The increasing amount of research questioning the efficacy of dichotic testing is occurring alongside a growing interest in, and clinical application of, auditory training for dichotic deficits (Moncrieff et al, 2016; Osisanya and Adewunmi, 2018). For example, Moncrieff et al (2017) investigated remediation of 125 children and adolescents aged 5-19 years diagnosed with amblyaudia and other binaural integration deficits. Amblyaudia is defined as an abnormally large asymmetry between the two ears during dichotic listening tasks with either normal or below normal performance in the dominant ear (Moncrieff et al, 2016). The participants completed four one-hour training sessions (40 minutes of training and 20 minutes of rest) with the Auditory Rehabilitation for Interaural Asymmetry (ARIA) program. ARIA training aims to improve nondominant ear performance, especially among participants whose interaural asymmetry was largest because of nondominant ear weakness. During ARIA, a clinician systematically adjusts the relative intensity of input to the two ears during dichotic listening exercises presented through sound-field speakers. Group statistics showed significant improvements in dichotic nondominant ear performance and reductions in interaural asymmetry between baseline dichotic test scores and test scores obtained following the fourth ARIA training session and again two or more months after training was completed.

The aim of the present study was to examine DDdT performance in a large population of children (with normal hearing and no known lesions of the CANS) referred for an auditory processing assessment, with the objective of evaluating the use of the dichotic advantage score as a method of differentiating children with true dichotic deficits from those who obtain low scores on a dichotic test for other reasons. A discussion on the statistical interpretation of EA in dichotic test data is also presented. 


\section{METHOD}

A pproval to use the data analyzed in this study was obtained from the participant's primary caregiver by an Australian Hearing audiologist before assessment.

\section{Participants}

Data collected from 101 children aged 6 years 3 months to 15 years 0 month (mean 9 years 6 months) who undertook a CAPD assessment by Australian Hearing were analyzed for this study. The Australian Hearing CAPD service specifically targets children experiencing listening deficits in noisy environments. All participants in the study had normal hearing, defined as equal to or better than $20-\mathrm{dB}$ HL at all octave frequencies between 500 and $4000 \mathrm{~Hz}$.

\section{Procedure}

All 101 participants completed the FR and diotic conditions of the DDdT. Ninety-three children also completed the number memory forward (NMF) and reverse (NMR) subtests of the Test of Auditory Processing-Third Edition (TAPS-3; Martin and Brownell, 2005). Administration of these tests is detailed in the following text. Completion of the directed ear conditions of the DDdT by the Australian Hearing service was optional and not analyzed for this study. Data were de-identified before submission by email by the audiologist. Gender was not recorded.

\section{DDdT}

The DDdT was administered with the use of a laptop computer and Senheisser HD215 headphones. Sound levels were calibrated using the procedure described in Cameron et al (2016a). The DDdT FR was completed before the diotic condition. Conditions were scored as percent correct and automatically converted to z-scores by the software using the formulas calculated in Cameron et al (2016a):

- Dichotic FR (FR LE, FR RE, and FR total): a set of two numbers were presented to the LE (e.g., 8 and 6) at the same time as two different numbers were presented to the RE (e.g., 1 and 3). The digits presented first to each ear overlapped in time, as did the digits presented second to each ear. Participants were asked to repeat back all four numbers in any order (e.g., 6, 8, 1, and 3). The $\mathrm{LE}$ and RE were scored separately. The average of the $\mathrm{LE}$ and RE scores-referred to as FR total-was also calculated. Five practice trials and twenty scored trials were presented.

- Diotic: A set of two numbers (e.g., 8 and 1) that were overlapping exactly in time, were presented to both ears. This was followed by another set of two numbers (e.g., 6 and 3) that were also overlapping exactly in time and were also presented to both ears. This resulted in four digits in total presented to both ears. Participants were asked to repeat back all four digits in any order. Five practice trials and twenty scored trials were presented.

The following difference scores are derived from the aforementioned DDdT conditions:

- Dichotic advantage: Dichotic FR total score minus diotic score.

- REA (FR): Dichotic FR RE score minus FR LE score.

\section{TAPS-3 NMF}

The NMF subtest of the TAPS-3 is a measure of verbal short-term memory. Digit sequences of increasing length were presented live-voice by the audiologist. The digits were presented at a rate of one digit per second. The client was tasked with repeating the digits back in the order they were heard. In accordance with the TAPS-3 instructions, if the digits were repeated in the correct order, without any additional digits inserted, a score of 2 was recorded. If an error was made in the order that the digits were repeated, then the item was scored as 1. If any digits were omitted or inserted during repetition, then a score of 0 was recorded. Testing was discontinued once three consecutive 0 point responses had been recorded. Results are reported as scaled scores.

\section{TAPS-3 NMR}

The NMR subtest of the TAPS-3 is a measure of verbal working memory. Digit sequences of increasing length were presented live-voice by the researcher, at a rate of one digit per second. The client was tasked with repeating the digits back to the audiologist in the reversed order. Scoring for the NMR is based on the same rules as used for the NMF, as discussed earlier.

\section{RESULTS}

Q tatistical analysis was performed using Statistica $\bigcirc$ version 10. All data are presented as z-scores to account for the effect of age on performance. DDdT data are reported for 101 participants in the clinical group. Data are also reported for the 93 participants who also completed the TAPS-3 NMF and NMR. Pairwise comparisons were conducted for comparison of DDdT and TAPS-3 results. The results from the clinical group are also compared with data from 112 TD children aged 7 years 0 month to 12 years 1 month (mean 9 years 3 months), published in Cameron et al (2016a,b). 


\section{Clinical and TD Group Comparison}

Group means and standard deviations (SDs) for the clinical group for the DDdT conditions and difference scores $(\mathrm{n}=101)$, as well for the TAPS-3 NMF and NMR ( $\mathrm{n}=93$ ), are provided in Table 1 . The previously published results for the TD children are provided for comparison for both the DDdT $(\mathrm{n}=112)$ and the NMF and NMR $(\mathrm{n}=50)$. The statistical significance of differences between the groups on each task is included in the table.

\section{DDdT}

In comparing dichotic and diotic performance between the groups, ANOVA revealed that the clinical group had significantly lower (poorer) age-adjusted scores than the TD group on both the DDdT FR total condition $\left[F_{(1,211)}=14.454, p=0.00019\right]$ and the diotic condition $\left[F_{(1,211)}=15.478, p=0.00011\right]$. However, there was no significant difference between the groups on the dichotic advantage measure $\left[F_{(1,211)}=0.000\right.$, $p=1.0000]$, indicating that the benefit obtained from dichotic listening compared with separating two simultaneously present sounds in the same ear was equivalent for the groups. Similarly, REA was not significantly different for the clinical and TD groups $\left[F_{(1,211)}=0.149\right.$, $p=0.70]$.

\section{Memory}

In comparing TAPS-3 NMF and NMR performance between the groups, ANOVA revealed that the clinical group had lower age-adjusted scores than the TD group on both the NMF $\left[F_{(1,141)}=10.784, p=0.001\right]$ and the $\operatorname{NMR}\left[F_{(1,141)}=3.802, p=0.01\right]$.

\section{Clinical Group Correlations}

Pearson product-moment correlation (pairwise) analysis was used to analyze whether correlations existed for the clinical children between various measures of $\operatorname{DDdT}(\mathrm{n}=101)$, as well as between the DDdT measures and memory tests $(n=93)$, as detailed in Table 2 . All measures were significantly correlated.

The correlations between the DDdT diotic condition and the various FR conditions were highly significant: FR LE $(r=0.87, p<0.00001)$, FR RE $(r=0.83, p<$ $0.00001)$, and FR total $(r=0.82, p<0.00001)$. Significant correlations were also found between the DDdT dichotic FR conditions and the NMF $(r=0.5-0.6$, $p<0.001)$ and the NMR $(r=0.2-0.5, p<0.023-$ 0.001 ). The DDdT diotic condition was also significantly correlated with the NMF $(r=0.60, p<0.001)$ and the $\mathrm{NMF}(r=0.49, p<0.001)$. Scatterplots of correlations between DDdT dichotic FR total and diotic z-scores, as well as between the NMF and the dichotic FR total and diotic z-scores, are provided in Figure 1A-C.

The scatterplot in Figure 2 provides a visualization comparison of the clinical and TD children from Cameron et al (2016a,b) in respect to the correlation between DDdT dichotic FR total and diotic performance. As with the clinical group, dichotic and diotic performance was highly correlated in the TD children $(r=0.69, p<0.00001)$.

\section{Multilinear Regression}

To investigate the contribution of memory and simultaneous segregation on variance in dichotic performance, multilinear regression with casewise deletion was performed for the 93 clinical children who had completed the DDdT conditions and the TAPS-3 NMR and NMF. The dependent variable was dichotic FR total z-score. Independent variables were DDdT diotic, NMF, and NMR z-score (see Table 3). These variables accounted for $68 \%$ of variation in dichotic performance $\left\{r^{2}=0.685\left[F_{(3,89)}=67.769, p<0.00001\right]\right\}$. There was no benefit of including NMR in the analysis. Diotic and NMF z-score alone still accounted for $68 \%$ of variation in dichotic performance $\left\{r^{2}=0.685\left[F_{(2,90)}=100.85\right.\right.$, $p<0.00001]\}$.

Table 1. Average Means and SDs for the Clinical Children on the DDdT Conditions and Advantage Measures $(n=101)$ and the TAPS-3 NMF and NMR Subtests $(n=93)$

\begin{tabular}{|c|c|c|c|c|c|c|c|}
\hline \multirow[b]{2}{*}{ Condition } & \multicolumn{3}{|c|}{ Clinical } & \multicolumn{3}{|c|}{$\mathrm{TD}$} & \multirow{2}{*}{$\begin{array}{c}\text { Group Difference } \\
\text { p-Value }\end{array}$} \\
\hline & $n$ & Mean z-Score & $\overline{S D}$ & $n$ & Mean z-Score & $\overline{S D}$ & \\
\hline$\overline{\mathrm{FR} \text { LE }}$ & 101 & -0.41 & 1.15 & 112 & -0.02 & 0.94 & 0.006 \\
\hline FR RE & 101 & -0.62 & 1.25 & 112 & -0.08 & 0.98 & $<0.001$ \\
\hline FR total & 101 & -0.62 & 1.24 & 112 & -0.03 & 0.99 & $<0.001$ \\
\hline Diotic & 101 & -0.48 & 1.11 & 112 & 0.09 & 0.98 & $<0.001$ \\
\hline Dichotic advantage & 101 & -0.10 & 0.91 & 112 & -0.10 & 0.90 & 1.00 \\
\hline FR REA & 101 & -0.08 & 1.15 & 112 & -0.03 & 0.90 & 0.70 \\
\hline NMF & 93 & -0.42 & 0.80 & 50 & 0.03 & 0.74 & 0.001 \\
\hline NMR & 93 & -0.11 & 0.76 & 50 & 0.23 & 0.72 & 0.01 \\
\hline
\end{tabular}

Notes: Results are compared with those for the TD children published in Cameron et al $(2016 a, b)$ on the DDdT $(n=112)$ and the NMF and NMR $(n=$ 50). Results reported as z-scores. The final column shows the significance of the difference between the group scores. 
Table 2. Pearson Product-Moment (Pairwise) Correlation Coefficient (r), Calculated from the Z-Scores of the Clinical Group on the Various DDdT Conditions $(n=101)$ and the TAPS-3 NMF and NMR $(n=93)$

\begin{tabular}{|c|c|c|c|c|c|c|}
\hline$\overline{\text { Test }}$ & FR LE & FR RE & FR Total & Diotic & NMF & NMR \\
\hline$\overline{\text { FR LE }}$ & 1.00 & $0.45^{\star \star}$ & $0.87^{\star \star}$ & $0.68^{\star \star}$ & $0.48^{\star \star}$ & $0.37^{\star \star}$ \\
\hline FR RE & & 1.00 & $0.82^{\star *}$ & $0.70^{\star *}$ & $0.51^{\star *}$ & $0.23^{*}$ \\
\hline FR total & & & 1.00 & $0.82^{\star *}$ & $0.60^{* *}$ & $0.38^{\star *}$ \\
\hline Diotic & & & & 1.00 & $0.60^{\star *}$ & $0.49^{\star \star}$ \\
\hline NMF & & & & & 1.00 & $0.46^{\star \star}$ \\
\hline NMR & & & & & & 1.00 \\
\hline
\end{tabular}

Notes: Significant correlations are marked with an asterisk $\left(^{*}<0.5 ;{ }^{* *}<0.001\right)$.

\section{Contribution of Random Measurement Error (RME) on Dichotic Performance}

The contribution of RME to dichotic performance in the clinical group was calculated using the SD of the DDdT dichotic FR total z-score test-retest differences of 0.70 for the 30 TD children published in Table 5 of Cameron et al (2016a). The formula used was $\left(\mathrm{SD}^{2} / 2\right) / T^{2}$, where $T^{2}(1.55)$ is the SD of the FR total test $\mathrm{z}$-scores for the 101 clinical children. The resulting variance in the FR total score was $16 \%$.

\section{Hypotheses from Study Results}

In respect to the variables that are explored in this study, it is hypothesized a child's ability to separate simultaneously presented stimuli (e.g., by using spectrotemporal processing) will impact both dichotic and diotic performance on the DDdT. Short-term memory and RME will also impact performance on both these tasks. The ability to separate different signals at each ear is unique to dichotic processing.

The contribution of the aforementioned factors to the variation in dichotic performance in the clinical children from this study has been estimated from the multilinear regression and calculation of RME described earlier. Figure 3 provides a visual representation of these contributions. After accounting for the influence of short-term memory, simultaneous segregation (diotic performance), and RME, only $16 \%$ of the variation in DDdT FR total z-scores can be attributed to the ability to separate different signals at each ear (dichotic processing). Although the clinical data analyzed for this study did not include a test of attention, this factor was found to be associated with variation in dichotic scores in Cameron et al (2016b) and in Stavrinos et al (2018), and cannot be ruled out as an additional variable contributing to dichotic test performance.

\section{Statistical Implications Impacting Interpretation of EA}

The analysis of the dichotic scores to this point has mainly focussed on the DDdT FR total score (i.e., LE plus RE). However, it is common clinically to place greater emphasis on the individual ear scores and on the difference in scores between ears. Figure 4 shows the difference in ear scores as a function of the FR total score for the clinical group. The solid regression line shows that, on average, REA increases as the total score decreases. When both ears have scores close to the ceiling of $100 \%$, EA scores are necessarily very small.

In interpreting EA scores, it is important to take into account the reliability of the data. The individual ear scores are less precise than the total score because the score for each ear is based on only 40 scored items rather than 80 . The difference score (i.e., EA) is even less precise because it is the difference between two such lower precision scores, and is thus affected by RME in both constituent scores. The magnitude of the random error variance affecting a score can be estimated, on average, from the binomial distribution statistics (Hagerman, 1976): Error variance $=P(1-P) / \mathrm{N}$, where $P$ is the proportion of items correct and $\mathrm{N}$ is the number of scored items. The error SD is the square root of the variance. For the clinical group assessed in this study, the average left- and right-ear scores were $77.7 \%$ and $82.6 \%$, respectively. The corresponding error SDs, with $\mathrm{N}=40$, are therefore $6.6 \%$ and $6.0 \%$, respectively. The error SD in the EA score is the square root of the sum of the corresponding variances, and is $8.9 \%$. Consequently, to be confident that an observed ear difference is anything other than typical REA plus the effect of RME, the REA would need to be greater than two SDs greater than or less than the mean. For the child with dichotic performance equal to the average of those in this clinical group, this criterion corresponds to REAs $>23 \%$ or $<-13 \%$. For those with poorer than average performance, this range widens because the binomialpredicted error variance increases as the true scores decrease from $100 \%$ to $50 \%$. This calculation can be repeated for different total scores, and the resulting limits of the scatter due to RME are shown as the dashed curves in Figure 4. These limits should encompass $95 \%$ of the data points if the scatter were to be caused entirely by RME. Ear-difference scores for 21 of the clinical children fall outside these limits. For these 21 children, it is possible that there are ear differences reliably 


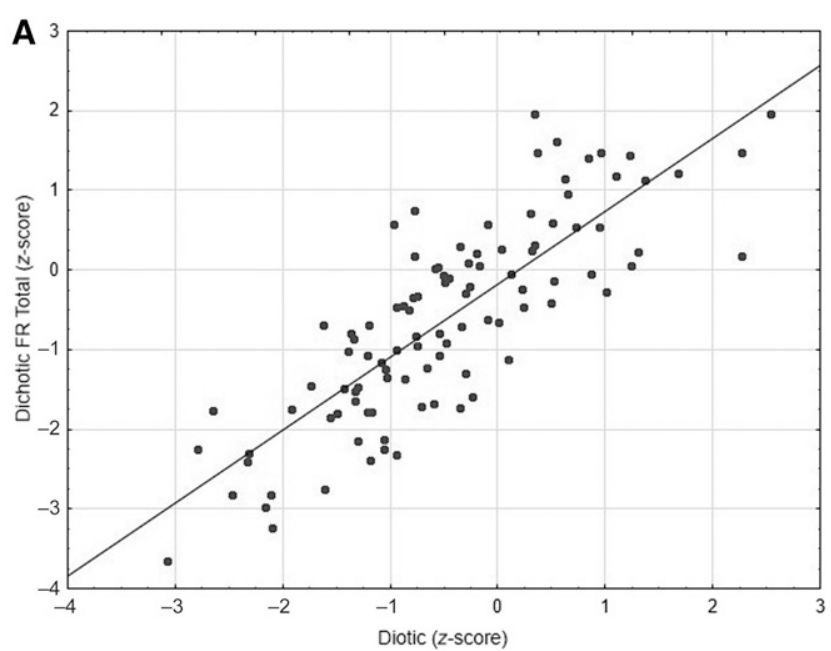

B

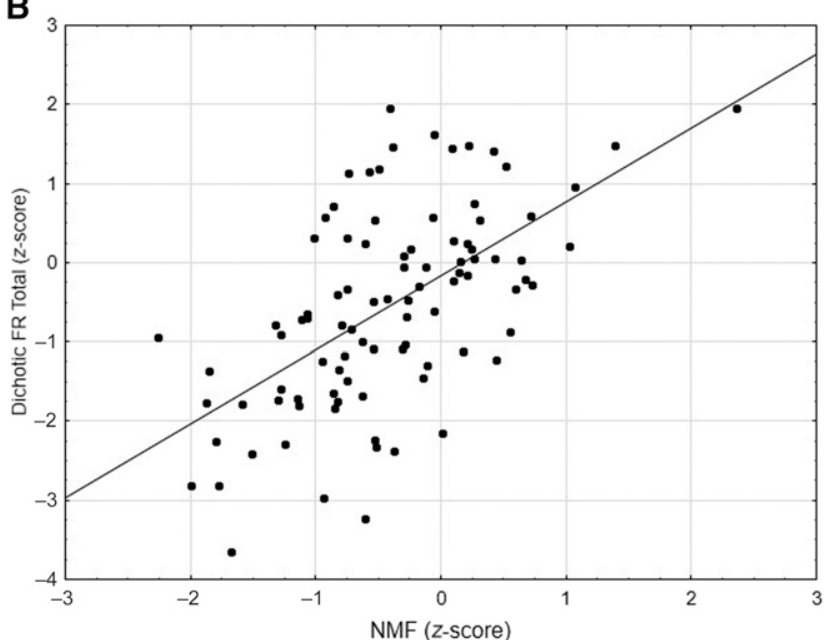

C

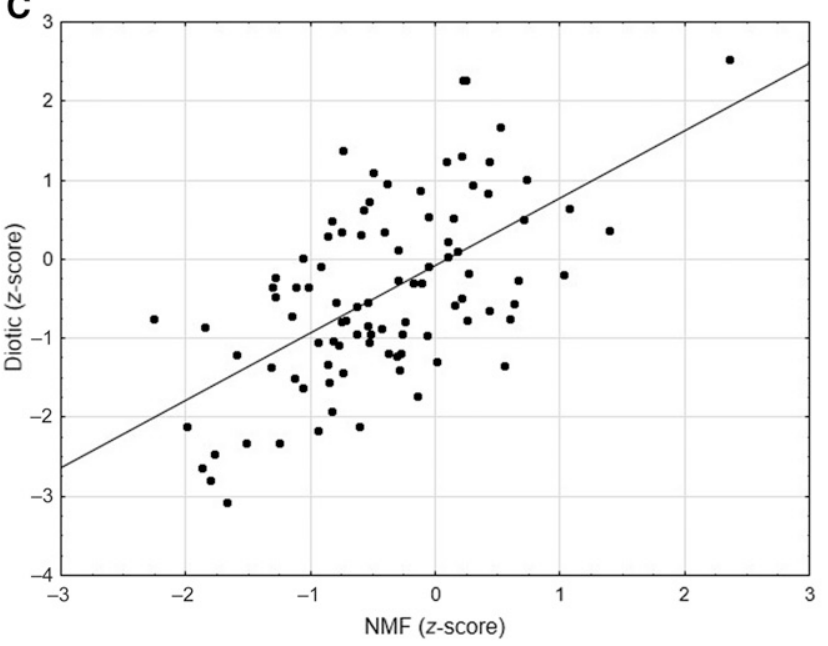

Figure 1. Scatterplots of children in the clinical group for (A) the DDdT FR total z-scores compared with the diotic z-scores $(\mathrm{n}=101)$; (B) FR total and TAPS-3 NMF ( $\mathrm{n}=93)$ and $(\mathrm{C})$ diotic and NMF $(\mathrm{n}=$ 93). The solid line represents least squares regression line.

different from average, albeit to a very small degree for most of them.

However, ear differences outside these limits set by unavoidable measurement error also occur for TD

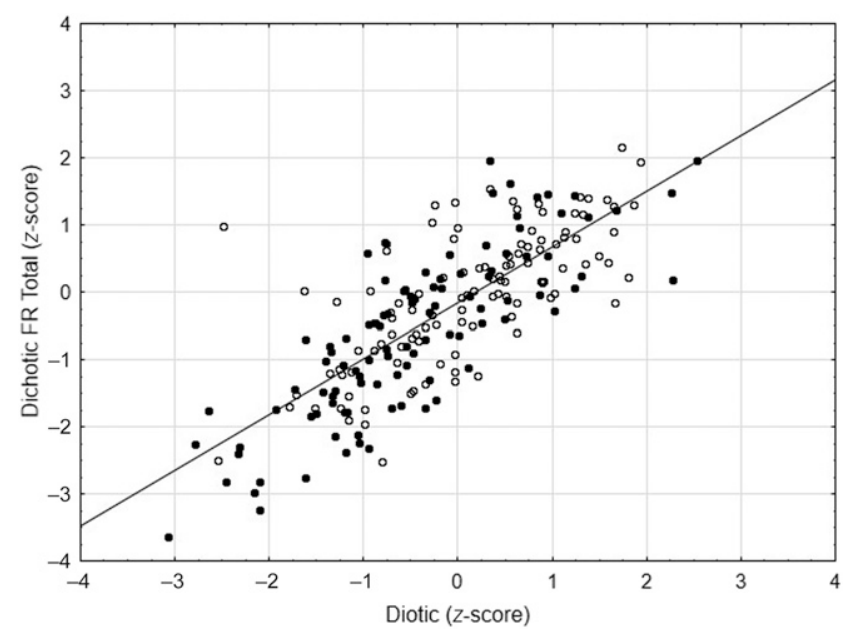

Figure 2. Scatterplot of the DDdT dichotic FR total and diotic z-scores for the 101 clinical children (solid circles) and the 112 TD children (open squares) from Cameron et al (2016a,b). The solid line represents least squares regression line.

children. Application of the formula in Cameron et al (2016a) for expressing the DDdT REA in population SD units (i.e., z-scores) revealed that only 7 of the 21 children had ear asymmetry of greater than two population SDs. For all but two of these, the magnitude of the ear asymmetry was less than \pm 2.5 SDs.

\section{DISCUSSION}

7 his study investigated the relationship between the various conditions of the DDdT and between the DDdT and TAPS-3 NMF and NMR in 101 children with normal hearing and no known lesions of the CANS, who received an auditory processing assessment by the Australian Hearing CAPD service. The impact of simultaneous segregation, memory, and RME on dichotic processing was calculated. The interpretation of EA was also discussed in light of statistical analysis and variation in performance. Finally, performance by the 101 children referred for auditory processing assessment by Australian Hearing centers was compared with data from 112 TD children from Cameron et al (2016a,b).

Just as found in the previous study with the TD children, the high correlation $(r=0.82, p<0.00001)$ between performance by the clinical group on the diotic condition and the FR total condition is striking. Both $\mathrm{NMF}$ and NMR results were significantly correlated with both dichotic FR total and diotic z-scores $(r=$ $0.4-0.6, p<0.001)$. Multilinear regression revealed that diotic processing (simultaneous segregation) and TAPS-3 NMF (short-term memory) accounted for $68 \%$ of the variation in dichotic processing, or the ability to separate different signals at each ear. RME accounted for a further $16 \%$ of the variation. This means that $84 \%$ of the variance in the FR total scores 
Table 3. Summary of Multilinear Regression Performed for the 93 Clinical Children Who Had Completed the DDdT and the TAPS-3 NMR and NMF

\begin{tabular}{|c|c|c|c|c|c|c|}
\hline Test z-score & Beta & SE of Beta & $b$ & SE of $b$ & $t(89)$ & $p$-Value \\
\hline Intercept & & & -0.09 & 0.08 & -1.12 & 0.27 \\
\hline DDdT diotic & 0.75 & 0.08 & 0.82 & 0.08 & 9.79 & $<0.0001$ \\
\hline NMF & 0.18 & 0.07 & 0.29 & 0.12 & 2.45 & 0.02 \\
\hline NMR & -0.08 & 0.07 & -0.12 & 0.11 & -1.09 & 0.28 \\
\hline
\end{tabular}

Notes: The dependent variable was dichotic FR total z-score. Beta values are standardized regression coefficients, and $b$ values are unstandardized.

is accounted for by factors that do not involve the perception of dichotic stimuli and only $16 \%$ of the total variance in FR total scores could potentially be accounted for by true differences in dichotic perception.

In Cameron et al (2016b), diotic performance and RME accounted for $83 \%$ of the total variance in dichotic FR total scores in a group of 50 TD children and 10 clinical children. We speculated that if a group with more aberrant dichotic perception due purely to true dichotic deficits were to be included, then this figure would decrease and we would see a greater amount of variation due to true dichotic perception. However, in our sample of 101 children referred for auditory processing assessment around Australia, this was not the case. Indeed, including memory in the multilinear regression explained even more variance in dichotic performance. Tests of attention were not performed by the Australian Hearing CAPD service, so we do not know how much variation in dichotic performance this additional factor could explain.

The incorporation of a diotic control condition in the DDdT provides the ability to calculate the ability to use dichotic processing to separate sounds arriving at each ear as a difference score, referred to as the dichotic advantage measure. In Figure 1A, which shows the relationship between DDdT diotic and dichotic FR total performance, it can be seen that all children with low dichotic scores also had poorer than average diotic scores. Just as found in the small study in Cameron et al (2016b), in the current large clinical study, it was found that although dichotic scores were lower for the clinical group than for the TD group, there was no difference between the groups in respect to their dichotic advantage scores $\left[F_{(1,211)}=0.0000, p=\right.$ $1.0000]$, indicating that, as a group, the clinical children are just as able to take advantage of dichotic cues as TD children.

As with other dichotic tests, calculating the difference in scores between the ears using the DDdT potentially gives useful information about the relative efficiency with which sounds input to each ear are processed. However, also as with other tests, measurement precision decreases with the number of test items and

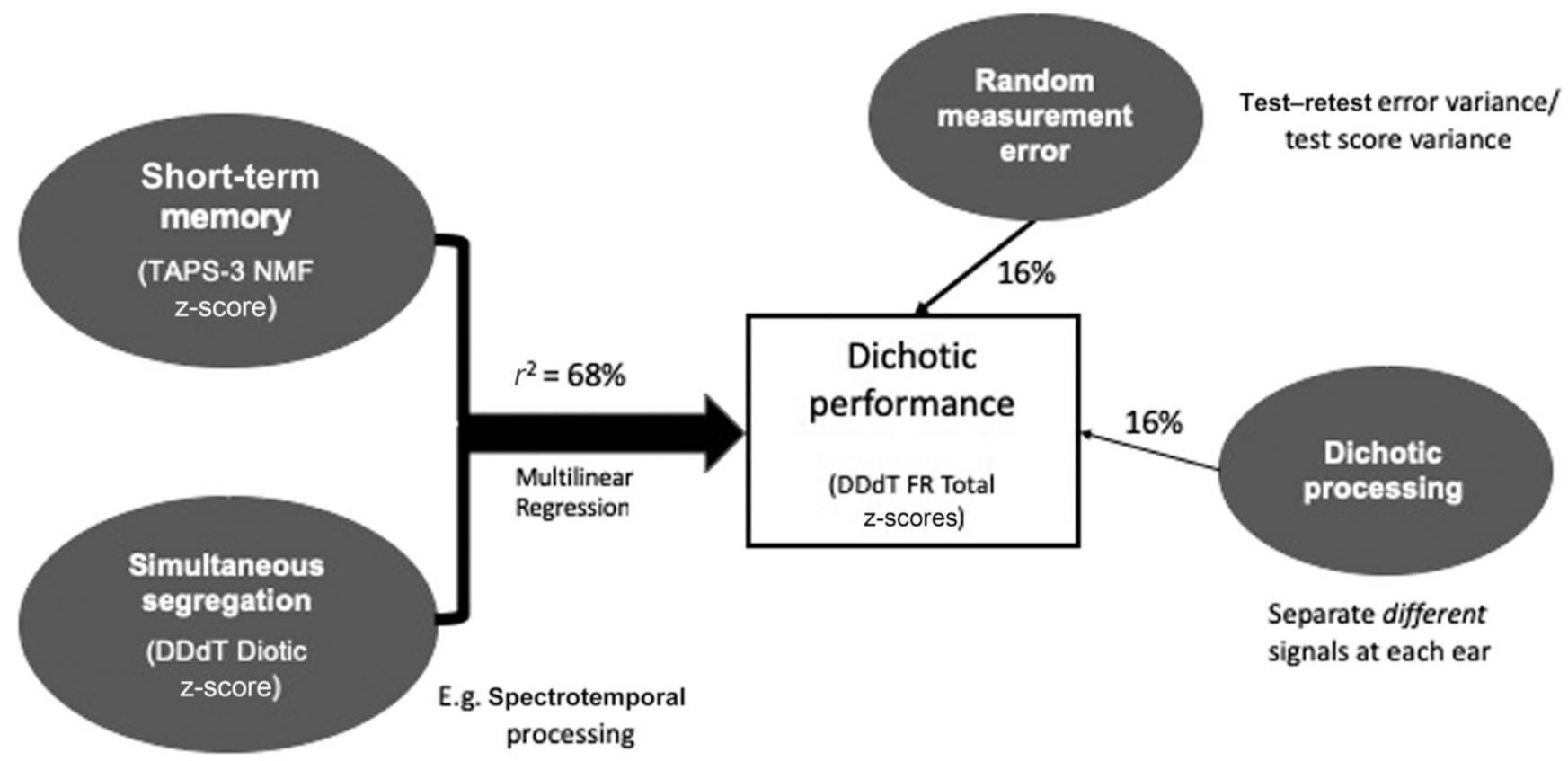

Figure 3. Diagram of impact of memory, simultaneous segregation, and RME on dichotic FR total z-scores in a clinical population $(\mathrm{n}=93)$. 


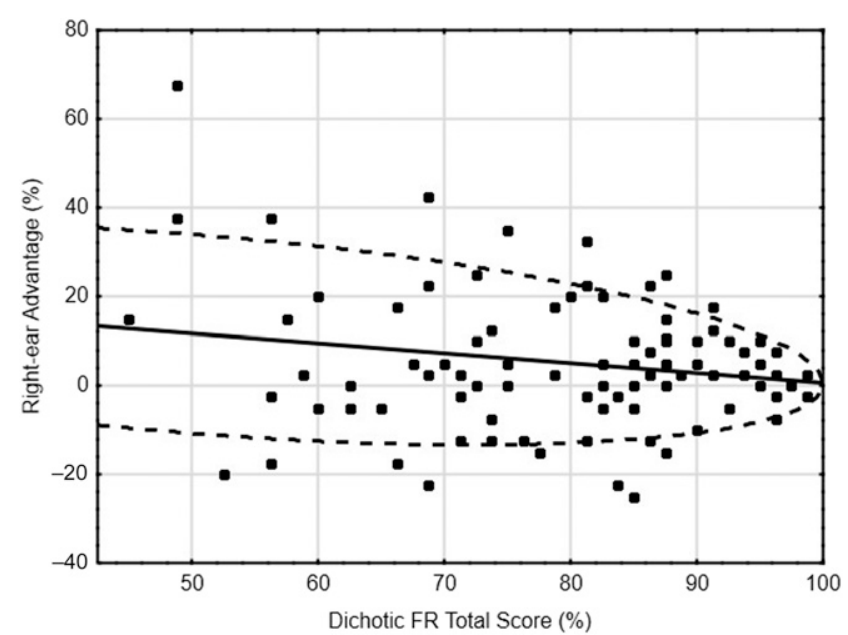

Figure 4. REA (in percentage points) as a function of the FR total score for the 101 children in the clinical group. The solid line shows the regression line, and the dashed line shows the $95 \%$ spread expected from random measurement error alone.

decreases further when two such scores are subtracted. For the children in this clinical sample, there were only 21 children with ear-difference scores outside the range that would be expected just on the basis of RME. For 14 of them, the extent of EA was still within the range covered by $95 \%$ of children drawn from a TD population. Only two of the remaining seven children had EA scores markedly outside the range found in a TD population. One of these had an unusually large REA, and the other had a left-EA. One feature of the DDdT test is that the results are automatically expressed in age-appropriate population SD units. On the basis of this measure applied to this large clinical sample, we conclude that ear asymmetry stronger than is common in the TD population, and that cannot be explained by RME, is relatively uncommon, even in a clinical population.

\section{CONCLUSION}

I $\mathrm{n}$ the discussion from Cameron et al (2016b), we stated that given the small number of clinical cases included in that study, it would be speculative to draw any conclusions at that point in time. However, based on this large clinical investigation and the analyses conducted, it appears that dichotic performance, at least on a two-pair dichotic digits test, is strongly associated with cognitive skills that are involved in perceiving and repeating simultaneously presented digits, even when there are not dichotic cues present. We conclude that the DDdT dichotic advantage score will provide a useful indicator of true dichotic processing ability. Children with a true dichotic deficit should have a markedly negative z-score on this measure. Furthermore, clinicians should be mindful of the impact of cognitive factors on dichotic tests. The hierarchical, decision tree, assessment structure recommended by Magimairaja and Nagaraj (2018) and applied clinically in Cameron et al (2015) is an added safeguard to ensure children who have issues such as memory scores outside normal limits are referred to the appropriate professional for further assessment and management and not erroneously diagnosed with an auditory processing disorder. Unfortunately, requiring memory ability to be within normal limits is not sufficient: The correlations between memory scores and dichotic perception reported here occurred despite almost all the memory scores being within the normal range $(-2<z<2)$. There is a need to quantitatively allow for the impact that cognitive performance has on individual auditory processing test scores.

In respect to the aforementioned, a discussion on causality and future research is warranted. Whereas the data and analyses presented here provide a strong argument as to the impact of cognitive functions on dichotic performance, a converse argument could be mounted that dichotic processing deficits result in low memory scores and not vice versa. To this end, future controlled double-blind studies are recommended whereby children diagnosed with a dichotic processing deficit are trained with either dichotic programs such as ARIA or memory training. Comparison of pre- and postremediation test scores on diagnostic tests of dichotic and memory processing will cast light on the direction of causality and assist clinicians in the future to determine the best assessment and management options for their clients.

Acknowledgments. The authors would like to thank the staff of Australian Hearing, who collected and shared the data for this study, in particular Alison King, Karin Gillies, and John Seymour who facilitated its collection. We would also like to thank the parents who gave permission for their child's test data to be used for research purposes.

\section{REFERENCES}

Cameron S, Dillon H. (2018) Deficit-specific diagnosis and remediation of auditory processing disorders. In: Geffner D, Ross-Swain D, eds. Auditory Processing Disorders: Assessment, Management and Treatment. 3rd ed. San Diego, CA: Plural Publications, 95-122.

Cameron S, Glyde H, Dillon H, King A, Gillies K. (2015) Results from a national central auditory processing disorder service: a "real world" assessment of diagnostic practices and remediation for CAPD. Semin Hear 36(4):216-236.

Cameron S, Glyde H, Dillon H, Whitfield J, Seymour J. (2016a) The Dichotic Digits difference Test (DDdT): development, normative data, and test-retest reliability studies part 1. J Am Acad Audiol 27(6):458-469.

Cameron S, Glyde H, Dillon H, Whitfield J. (2016b) Investigating the interaction between dichotic deficits and cognitive abilities using the Dichotic Digits difference Test (DDdT) part 2. J Am Acad Audiol 27(6):470-479. 
Cameron S, Glyde H, Seymour J, Dillon H. (2013) Dichotic Digits Difference Test (DDdT) (Research Version) [Computer Software]. Sydney, NSW: National Acoustic Laboratories.

Dillon H, Cameron S, Glyde H, Wilson W, Tomlin D. (2012) Opinion: re-designing the process of assessing people suspected of having central auditory processing disorders. J Am Acad Audiol 23(2): 97-105.

Fischer ME, Cruickshanks KJ, Nondahl DM, Klein BEK, Klein R, Pankow JS, Tweed TS, Dalton DS, Paulsen AJ. (2017) Dichotic digits test performance across the ages: results from two large epidemiologic cohort studies. Ear Hear 38(3):314-320.

Hagerman B. (1976) Reliability in the determination of speech discrimination. Scand Audiol 5:219-228.

Hiscock M, Kinsbourne M. (2011) Attention and the right-ear advantage: what is the connection? Brain $\operatorname{Cogn} 76: 263-275$.

Hurley R, Musiek F. (1997) Effectiveness of three central auditory processing (CAP) tests in identifying cerebral lesions. J Am Acad Audiol 8(4):257-262.

Kelley KS, Littenberg B. (2018) Structured review of dichotic tests of binaural integration: clinical performance in children. $A m J$ Audiol 27:156-166.

Magimairaja BM, Nagaraj NK. (2018) Working memory and auditory processing in school-age children. Lang Speech Hear Serv Sch 49:409-423.

Martin N, Brownell R. (2005) Test of Auditory Processing Skills. 3rd ed. Novato, CA: Academic Therapy Publications.

Martin J, Jerger J, Mehta J. (2007) Divided-attention and directed-attention listening modes in children with dichotic deficits: an event-related potential study. J Am Acad Audiol 18(1):34-53.

Moncrieff D, Keith W, Abramson M, Swann A. (2016) Diagnosis of amblyaudia in children referred for auditory processing assessment. Int J Audiol 55(6):333-345.
Moncrieff D, Keith W, Abramson M, Swann A. (2017) Evidence of binaural integration benefits following ARIA training for children and adolescents diagnosed with amblyaudia. Int $J$ Audiol 56(8): $580-588$.

Moore R, Sieswerda SL, Grainger MM, Bowling A, Smith N, Perdew A, Eichert S, Alston S, Hilbert LW, Summers L, Lin L, Hunter LL. (2018) Referral and diagnosis of developmental auditory processing disorder in a large, United States hospital-based audiology service. J Am Acad Audiol 29(5):364-377.

Musiek M. (1983) Assessment of central auditory dysfunction: the dichotic digit test revisited. Ear Hear 5(2):79-83.

Musiek M, Chermak G, Weihing J, Zappulla M, Nagle S. (2011) Diagnostic accuracy of established central auditory processing test batteries in patients with documented brain lesions. $J$ Am Acad Audiol 22(6):342-358.

Osisanya A, Adewunmi A. (2018) Evidence-based interventions of dichotic listening training, compensatory strategies and combined therapies in managing pupils with auditory processing disorders. Int J Audiol 57(2):115-123.

Schmithorst VJ, Farah R, Keith RW. (2013) Left ear advantage in speech-related dichotic listening is not specific to auditory processing disorder in children: a machine-learning fMRI and DTI study. Neuroimage Clin 3:8-17.

Stavrinos G, Iliadou VM, Edwards L, Sirimanna T, Bamiou DE. (2018) The relationship between types of attention and auditory processing skills: reconsidering auditory processing disorder diagnosis. Front Psychol 9(34):1-13.

Weihing J, Atcherson SR. (2014) Dichotic listening tests. In: Chermak GD, Musiek FE, eds. Handbook of Central Auditory Processing Disorder. Auditory Neuroscience and Diagnosis. Vol. I. San Diego, CA: Plural Publishing, 369-404.

Westerhausen R, Hugdahl K. (2008) The corpus callosum in dichotic listening studies of hemispheric asymmetry: a review of clinical and experimental evidence. Neurosci Biobehav Rev 32:1044-1054. 